Meta

Journal des traducteurs

Translators' Journal

\title{
Lexique français-anglais de la culture des plantes d'intérieur
}

\section{France Guertin}

Volume 33, numéro 4, décembre 1988

Symposium AILA 1987, Sydney

URI : https://id.erudit.org/iderudit/002979ar

DOI : https://doi.org/10.7202/002979ar

Aller au sommaire du numéro

Éditeur(s)

Les Presses de l'Université de Montréal

ISSN

0026-0452 (imprimé)

1492-1421 (numérique)

Découvrir la revue

Citer cet article

Guertin, F. (1988). Lexique français-anglais de la culture des plantes

d'intérieur. Meta, 33(4), 560-565. https://doi.org/10.7202/002979ar d'utilisation que vous pouvez consulter en ligne.

https://apropos.erudit.org/fr/usagers/politique-dutilisation/ 


\section{LEXIQUE FRANÇAIS-ANGLAIS DE LA CULTURE DES PLANTES D'INTÉRIEUR*}

à l'étroit dans son pot aquiculture

arrosage

arrosage par capillarité

asexué,ée

bassinage

bonsaï

bonzaï

bouturage

bouture

bouture de feuille

bouture de racine pot-bound, pot bound

hydroponics, soilless culture, water culture, nutriculture watering

wick-watering

asexual, vegetative

misting

bonsai, penjing

bonsai, penjing

cutting, cuttage

cutting

leaf cutting, leaf-cutting

root cutting, root-cutting 


\begin{tabular}{|c|c|}
\hline $\begin{array}{l}\text { bouture de tige } \\
\text { bulbe }\end{array}$ & $\begin{array}{l}\text { stem cutting } \\
\text { bulb }\end{array}$ \\
\hline caisse de Ward & terrarium, Wardian case \\
\hline compost & compost \\
\hline culture hydroponique & $\begin{array}{l}\text { hydroponics, soilless culture } \\
\text { water culture, nutriculture }\end{array}$ \\
\hline dessèchement & wilting, wilt \\
\hline division & division \\
\hline dont le pot est trop petit & pot-bound, pot bound \\
\hline éboutonnage & disbudding \\
\hline empotage & potting \\
\hline empotement & potting \\
\hline engrais & fertilizer \\
\hline entrenoeud & internode \\
\hline entre-noeud & internode \\
\hline état dormant & dormancy \\
\hline fanaison & wilting, wilt \\
\hline fertilisation & fertilization, feeding \\
\hline feuille & leaf \\
\hline flétrissure & wilting, wilt \\
\hline fongicide & fungicide \\
\hline forçage & forcing \\
\hline génération & reproduction, propagation \\
\hline génération sexuée & breeding, sexual reproduction \\
\hline greffage & grafting \\
\hline greffe & grafting \\
\hline humus & humus \\
\hline hydroculture & $\begin{array}{l}\text { hydroponics, soilless culture, } \\
\text { water culture, nutriculture }\end{array}$ \\
\hline hydroponie & $\begin{array}{l}\text { hydroponics, soilless culture, } \\
\text { water culture, nutriculture }\end{array}$ \\
\hline imbibé d'eau & waterlogged \\
\hline imprégné d'eau & waterlogged \\
\hline insecticide & insecticide \\
\hline jardinage sans sol & $\begin{array}{l}\text { hydroponics, soilless culture, } \\
\text { water culture, nutriculture }\end{array}$ \\
\hline jardin en bouteille & terrarium, Wardian case \\
\hline marcottage & layering \\
\hline marcottage aérien & $\begin{array}{l}\text { air layering, air-layering, Chinese } \\
\text { layering, mossing, marcottage }\end{array}$ \\
\hline marcottage en l'air & $\begin{array}{l}\text { air layering, air-layering, Chinese } \\
\text { layering, mossing, marcottage }\end{array}$ \\
\hline marcotte & layering \\
\hline marcotte aérienne & $\begin{array}{c}\text { air layering, air-layering, Chinese } \\
\text { layering, mossing, marcottage }\end{array}$ \\
\hline motte & root ball \\
\hline multiplication & $\begin{array}{l}\text { propagation, vegetative propagation } \\
\text { asexual reproduction }\end{array}$ \\
\hline ation ase & propagation, vegetative propagation, \\
\hline
\end{tabular}


multiplication végétative

mutation
noud
orifice de drainage
penjing
période de repos

PH

photosynthèse

pinçage

pincement

plante acidophile

plante calcifuge

plante d'appartement

plante de jour court

plante de jour long

plante de jours courts

plante de jours longs

plante de maison

plante de nuit courte

plante de nuit longue

plante d'intérieur

plante grasse

plante neutre

plante succulente

potentiel d'hydrogène

potentiel hydrogène

racine

rejet

rempotage

repiquage

repos végétatif

reproduction

reproduction asexuée

reproduction sexuée

respiration

ressuyage

rhizome

sexué, ée

sexuel, elle

sol

solution fertilisante

stomate

surfaçage

terrarium

terre

terreau de feuilles asexual reproduction

propagation, vegetative propagation, asexual reproduction

\section{mutation}

node, leaf node, leaf axil, growth bud

drainage hole, bottom hole

penjing, bonsai

rest period, rest-period,

$p H$

resting period

photosynthesis

pinching, pinching back

pinching, pinching back

acid-loving plant

calcifuge (plant), lime-hater (plant)

house plant, houseplant, indoor plant

short-day plant

long-day plant

short-day plant

long-day plant

house plant, houseplant, indoor plant

long-day plant

short-day plant

house plant, houseplant, indoor plant succulent

day-neutral plant

succulent

$p H$

pH

root

shoot

shifting, potting on, repotting

pricking off, pricking out

rest period, rest-period, resting period reproduction, propagation

propagation, vegetative propagation,

asexual reproduction

breeding, sexual reproduction

respiration

drying

rhizome, rootstock

sexual

sexual

soil

fertilizer

stomata

top-dressing

terrarium, Wardian case

soil

leaf mold, leafmold, leaf mould 


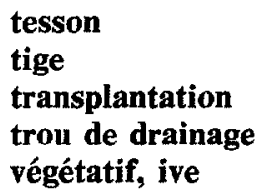

crock

stem

pricking off, pricking out drainage hole, bottom hole vegetative, asexual

\section{LEXIQUE ANGLAIS-FRANÇAIS}

acid-loving plant air layering

air-layering

asexual

asexual reproduction

bonsai

bottom hole

breeding

bulb

calcifuge (plant)

Chinese layering

compost

crock

cuttage

cutting

cutting

day-neutral plant

disbudding

division

dormancy

drainage hole

drying

feeding

fertilization

fertilizer

forcing

fungicide

grafting

growth bud

houseplant

house plant

humus

hydroponics plante acidophile

marcottage aérien, marcottage en l'air, marcotte aérienne

marcottage aérien, marcottage en l'air, marcotte aérienne

asexué,ée, végétatif,ive

multiplication, multiplication végétative, reproduction asexuée, multiplication asexuée

bonsaï, bonzaï, penjing

trou de drainage, orifice de drainage reproduction, génération sexuée, reproduction sexuée

bulbe

plante calcifuge

marcottage aérien, marcottage en l'air, marcotte aérienne

compost

tesson

bouturage, bouture

bouturage, bouture

bouture

plante neutre

éboutonnage

division

dormance, état dormant

trou de drainage, orifice de drainage

ressuyage

fertilisation

fertilisation

engrais, solution fertilisante

forçage

fongicide

greffe, greffage

noud

plante d'intérieur, plante d'appartement, plante de maison

plante d'intérieur, plante d'appartement, plante de maison

humus

culture hydroponique, hydroponie, 


indoor plant
insecticide
internode
layering
leaf
leaf axil
leaf cutting
leaf-cutting
leaf mold
leafmold
leafmould
leaf node
lime-hater (plant)
long-day plant
marcottage
misting
mossing
mutation
node
nutriculture

penjing

PH, pH

photosynthesis

pinching

pinching back

pot bound

pot-bound

potting

potting on

pricking off

pricking out

propagation

\section{propagation \\ repotting \\ reproduction \\ respiration \\ resting period}

hydroculture, aquiculture, jardinage sans sol plante d'intérieur, plante d'appartement, plante de maison

insecticide

entrenœud, entre-noud

marcottage, marcotte

feuille

noud

bouture de feuille

bouture de feuille

terreau de feuilles

terreau de feuilles

terreau de feuilles

noud

plante calcifuge

plante de jour long, plante de jours

longs, plante de nuit courte

marcottage aérien, marcottage en l'air, marcotte aérienne

bassinage

marcottage aérien, marcottage en l'air, marcotte aérienne

mutation

noud

aquiculture, culture hydroponique,

hydroponie, hydroculture,

jardinage sans sol

penjing, bonsaï, bonzaï

$p H$, potentiel d'hydrogèene, potentiel hydrogène

photosynthèse

pincement, pinçage

pincement, pinçage

à l'étroit dans son pot, dont le pot est trop petit

à l'étroit dans son pot, dont le pot est trop petit

empotage, empotement

rempotage

repiquage, transplantation

repiquage, transplantation

multiplication, multiplication végétative, reproduction asexuée, multiplication asexuée

reproduction

rempotage

reproduction, génération

respiration

repos végétatif, période de repos 


rest period
rest-period
rhizome
root
root ball
root cutting
root-cutting
rootstock
sexual
sexual reproduction
shifting
shoot
short-day plant
soil
soilless culture

stem
stem cutting
stomata
succulent
terrarium
top-dressing
vegetative
vegetative propagation

\section{Wardian case \\ water culture \\ watering \\ waterlogged \\ wick-watering \\ wilt \\ wilting}

repos végétatif, période de repos repos végétatif, période de repos rhizome

racine

motte

bouture de racine

bouture de racine

rhizome

sexué,ée, sexuel,elle

reproduction, génération sexuée, reproduction sexuée

rempotage

rejet

plante de jour court, plant de jours courts, plante de nuit longue

sol, terre

aquiculture, culture hydroponique,

hydroculture, hydroponie,

jardinage sans sol

tige

bouture de tige

stomate

plante grasse, plante succulente terrarium, caisse de Ward, jardin en bouteille

surfaçage

asexué,ée, végétatif,ive

multiplication, multiplication

végétative, reproduction asexuée, multiplication asexuée

terrarium, caisse de Ward, jardin en bouteille

aquiculture, culture hydroponique, hydroponie, hydroculture, jardinage sans sol

arrosage

imprégné d'eau, imbibé d'eau

arrosage par capillarité

fanaison, flétrissure, dessèchement fanaison, flétrissure, dessèchement

FRANCE GUERTIN

Note

* Liste tirée de Lexique bilingue de la culture des plantes d'intérieur, Université de Montréal, travail dirigé. 